\title{
CONTRIBUTION A L'ÉTUdE DE LA RÉFRACTOMÉTRIE DES LACTO-SÉRUMS
}

\author{
par A. LATAIX, \\ Docteur-Vétérinaire Capitaine
}

\begin{abstract}
Travail du Laboratoire de Chimie-Physique du Professeur A. Boutaric
(Faculté des Sciences de l'Université de Dijon)
\end{abstract}

(Suite et fin)

RÉSULTATS EXPÉRIMENTAUX DE LA SYNTHÈSE DES LACTO-SÉRUMS

10 Matières salines: Dans leur ensemble, les matières salines mises successivement en solution, ont augmenté l'indice de l'eau distillée dans des limites variant entre 0,0014 et 0,0016

$$
\text { (moyenne : } 0,00145 \text { ) }
$$

Le chlorure de sodium a fait varier l'indice de 0,0003 à 0,0004 (moyenne : 0,00036 )

Le sulfate de potassium : de 0,0001 ;

Le citrate tri-calcique : de 0,0003 à 0,0004 (moyenne : 0,00033 );

Le citrate tri-potassique : de 0,0001 ;

Le citrate tri-magnésien : de 0,0001 .

Les citrates dans leur ensemble, ont apporté à l'indice une augmentation variant de 0,0005 à 0,0006 (moyenne : 0,00053 ).

Le phosphate mono-potassique a fait varier l'indice de 0,0001 à 0,0002 (moyenne : 0,00012).

Le phosphate bi-potassique : de 0,0001 à 0,0002 (moyenne : 0,00014 ).

Les phosphates, dans leur ensemble, ont augmenté l'indice de 0,0002 à 0,0003 (moyenne : 0,00026).

Le bicarbonate de sodium a fourni une augmentation de 0,0001 ; il en a été de même pour l'urée.

$2^{\circ}$ Lactose : Suivant les laits envisagés, l'augmentation apportée à l'indice de réfraction par le lactose a varié entre 0,0069 et 0,0076 (moyenne : 0,0073).

Tous ces résultats sont rassemblés en un tableau récapitulatif de la synthèse de nos lacto-sérums.

En résumé, l'indice total moyen de ces sérums est la somme des indices de l'eau distillée, des matières salines en solution vraie $e t$ surtout du lactose:

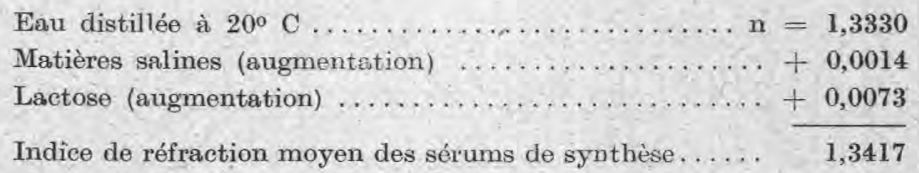

Le lactose est évidemment le gros composant des matières en solution dans le lacto-sérum. Il en représente, en poids, plus des 5/6 et l'augmen- 
tation apportée par elles à l'indice de réfraction de l'eau distillée est influencé dans la même proportion (plus des 5/6) par la lactose.

Il convient de remarquer que les indices de réfraction totaux de nos sérums se classent exactement suivant la richesse en lactose des laits auxquels ils se rapportent; déterminer l'indice de réfraction d'un lactosérum, c'est donc, d'une manière indirecte, évaluer la teneur du lait en lactose, du moins avec un sérum libre d'albumines.

Dans l'ensemble, les indices trouvés pour nos sérums de synthèse différent, en moins, de 0,0001 , environ, de ceux fournis par les sérums d'ultrafiltration. Cet écart reste dans la limite des erreurs d'expérience.

Nous ne saurions aspirer, au surplus, à une exactitude absolue dans les recherches synthétiques que nous avons entreprises.

Nous ne prétendons pas à une précision rigoureuse, nous espérons simplement avoir donné une image de la vérité aussi peu déformée que possible.

\section{Etablissement d'une classification des lacto-sérums. \\ Etude critique de leur valeur pratique. Choix d'une méthode.}

Des recherches qui précèdent, nous conclurons, que d'accord avee les résultats des travaux de Connalba, de Wiegner et du Professeur Ch. Poroher, le lacto-sérum idéal sera celui qui ne renfermera pas d'albumines et dont la composition et le volume se rapprocheront le plus de la phase solution vraie du lait qui, d'après les travaux de ces auteurs, est la plus constante des différentes phases (émulsoïde et colloïdale) de cette sécrétion.

N'oublions pas le point de vue pratique de la réfractométrie des sérums de lait : déceler le mouillage de la façon la plus sensible et la plus nette.

Pour se rapprocher de cet idéal, il faudra employer le plus petit volume possible de coagulant et utiliser une méthode qui, physiquement ou chimiquement, élimine les albumines, afin d'utiliser au maximum, pour la recherche du mouillage, la différence qui existe entre la réfraction de la phase solution vraie et celle de l'eau.

Et, pour simplifier la question, avec L. PANCHAUD, nous pourrons appeler sensibilité de la méthode, la différence qui existe entre la réfraction normale minimum $R$ du sérum et celle $R_{0}$ de l'eau en volume égal à celui du lait employé pour obtenir le sérum et contenant la même proportion de coagulant que le lait traité.

L'influence d'un faible mouillage sera d'autant plus marquée que $\mathbf{R}-\mathbf{R}_{\mathrm{o}}$ sera plus grand. 
Nous classerons les méthodes de préparation des lacto-sérums en deux catégories d'après leur mode d'obtention :

A Lacto-sérums rréparés à froid.

B Lacto-sérums préparés à chaud.

A LACTO-SÉRUMS PRÉPARÉS A FROID.

10 Sérum spontané.

(Utz-Matthes et Müller.)

Mode opératoire. On laisse le lait s'aigcir naturellement dans un flacon fermé, jusqu'à ce que la coagulation se produise. On filtre le sérum. Comme la coagulation est lente à se produire, on peut la hâter en portant le flacon au bain-marie à $35^{\circ}-40^{\circ} \mathrm{C}$.

Suivant la saison et le degré d'acidité de l'échantillon employé, la durée de la coagulation varie de 6 à 18 heures.

Le sérum obtenu est généralement limpide et contient en solution la lactalbumine et la lactoglobuline.

C'est un sérum à l'acide lactique formé aux dépens du lactose :

$$
\mathrm{C}^{12} \mathrm{H}^{22} \mathrm{O}^{11}+\mathrm{H}^{2} \mathrm{O}=4 \mathrm{C}^{3} \mathrm{H}^{6} \mathrm{O}^{3}
$$

\section{1 molécule de lactose hydraté 4 molécules d'acide lactique}

Mais, en réalité, selon la nature des germes préexistants dans le lait, il se forme de l'acide lactique, en proportion plus ou moins grande, il y a également production d'acides carbonique, propionique, butyrique; en outre l'albumine se transforme en ses sous-produits, les microbes habituels du lait arrêtant habituellement leur action au stade peptone.

Dans ces contitions, la composition du sérum spontané n'est jamais comparable d'un échantillon à l'autre.

$$
\left.\begin{array}{l}
\mathrm{R}=1,3425-1,3436 \text { à } 20^{\circ} \mathrm{C} . \\
\mathrm{R}_{0}=1,3330 \\
i d .
\end{array}\right\} \text { Sensibilité }=0,0095 \text { environ }
$$

Critique: Cette méthode n'est pas à recommander, ses résultats n'étant pas contrôlables ni toujours identiques; de plus, l'obtention du lacto-sérum est de trop longue durée.

\section{Sérum à 1'asaprol (Baïer et Neumann)}

L'asaprol ou abrastol est une combinaison calcique du dérivé * monosulfoné du naphtol $\beta$.

C'est un naphtylsulfate de chaux de formule :

$$
\begin{aligned}
& \mathrm{HO}-\mathrm{C}^{10} \mathrm{H}^{6}-\mathrm{SO}^{3}>\mathrm{Ca}+3 \mathrm{H}^{2} \mathrm{O} \\
& \mathrm{HO}-\mathrm{C}^{10} \mathrm{H}^{6}-\mathrm{SO}^{3}
\end{aligned}
$$

Mode opératoire: On agite $15 \mathrm{~cm}^{3}$ de lait avec $15 \mathrm{~cm}^{3}$. de solution d'asaprol ( $30 \mathrm{gr}$. d'asaprol et $55 \mathrm{gr} .8$ d'acide citrique cristallisé dissout dans un litre d'eau distillée). On sépare par filtration le coagulum de caséine et de matière grasse du sérum. 
Le sérum est très limpide.

La solution d'abrastol et le sérum sont à peu près isoréfringents

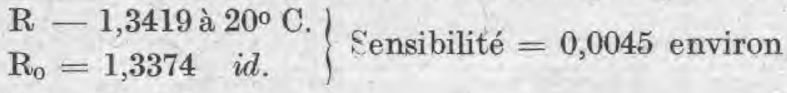

\section{Critique :}

Avantages: Méthode rapide, sérum très clair;

Inconvénients: Procédé peu sensible, le lait étant dilué par le réactif dans la proportion de $100 \%$. De plus, la solution d'abrastol ne se conserve pas et noircit très vite.

\section{$3^{\circ}$ Sérum au sulfate de cuivre}

\section{(H. Lythgoe.)}

Mode opératoire: $40 \mathrm{~cm}^{3}$ de lait sont traités par $10 \mathrm{~cm}^{3}$ de solution de sulfate de cuivre $(72,5 \mathrm{gr}$. par litre). On agite, on laisse reposer quelques minutes et on filtre; si le liquide passe trouble, on le verse à nouveau sur le filtre. Le sérum clair est soumis à l'examen réfractométrique.

(La solution de sulfate de euivre doit donner à $20^{\circ} \mathrm{C}$. un indice de réfraction $=1,3412$.)

$$
\begin{aligned}
& \mathrm{R}=1,3419 \text { à } 20^{\circ} \mathrm{C} . \\
& \mathrm{R}_{0}=1,3348 \quad \text { id. }
\end{aligned}\{\text { Sensibilité }=0,0071 \text { environ }
$$

Critique: Avantages: Préparation rapide et à froid, sérum très clair.

Inconvénients: Méthode peu sensible, le lait étant dilué par le réactif dans la proportion de $25 \%$.

\section{$4^{0}$ Tétrasérum I \\ (Pfyl et Turnau.)}

Mode opératoire: On mélange bien, en agitant fortement, $50 \mathrm{~cm}^{3} \mathrm{de}$ lait avec $5 \mathrm{~cm}^{3}$ de tétrachlorure de carbone, dans un flacon bien bouché ; on ajoute ensuite $1 \mathrm{~cm}^{3}$ d'acide acétique à $20 \%$. On agite à nouveau pendant quelques minutes. On centrifuge ou on filtre.

Le tétrachlorure de carbone est pratiquement insoluble dans l'eau, il est done sans influence sur la réfraction, il facilite la filtration en retenant dans le coagulum le maximum de graisse.

Le sérum obtenu est très clair, même lorsque le lait est fortement acide.

$$
\left.\begin{array}{l}
R \text { à } 20^{\circ} \mathrm{C} \text {., varie entre } 1,3433-1,3444 \\
R_{0}=1,3333
\end{array}\right\} \begin{gathered}
\text { Sensibilité }=0,0100 \\
\text { environ }
\end{gathered}
$$

Si le lait a été préalablement bouilli, l'indice de réfraction peut s'abaisser de 0,0003 à 0,0012 par suite de la précipitation de la lactalbumine et de la lactoglobuline. On peut donc conclure à un mouillage. Pour éliminer toutes les causes d'erreur il conviendra, dans ces cas douteux, de s'assurer que le lait n'a pas été bouilli ou additionné de lait bouilli. La réfraction du sérum, après ébullition du lait cru devant s'abaisser normalement de 0,0003 à 0,0012 . 
Critique: Avantages: Préparation à froid, sérum très limpide même quand le degré d'acidité du lait est élevé.

Filtration aisée.

Inconvénients: Le sérum renferme les albumines.

\section{$5^{\circ}$ Sérum chloruromercurique}

\section{(Ambuhl et Weiss.)}

Préparation du coagulant: Dans un ballon jaugé de $100 \mathrm{~cm}^{3}$, on met 125 gr. de bichlorure de mercure chimiquement pur. On ajoute, par petites quantités, jusqu'à dissolution, en chauffant sur une petite flamme, de l'acide chlorhydrique concentré $(\mathrm{d}=1,184)$. On refroidit à $15^{\circ} \mathrm{C}$. et on complète à $100 \mathrm{~cm}^{3}$ avec l'acide chlorhydrique concentré.

Le contrôle de la solution coagulante est fait en opérant un essai à blanc : à $30 \mathrm{~cm}^{3}$ d'eau distillée, on ajoute $0,3 \mathrm{~cm}^{3}$ de coagulant. On agite. L'indice de réfraction du mélange ainsi obtenu doit être à $17^{\circ} 5 \mathrm{C}$., de 20 divisions du réfractomètre à immersion de Zeiss $(n=1,3351)$.

Mode opératoire: A $30 \mathrm{~cm}^{3}$ de lait, ajouter $0,3 \mathrm{~cm}^{3}$ de coagulant. Agiter vigoureusement. Filtrer. L'indice de réfraction d'un lait normal est de 1,3436 .

Le sérum est très limpide, quelle que soit l'acidité du lait; il ne renferme ni lactalbumine ni lactoglobuline.

$$
\left.\begin{array}{l}
\mathrm{R}=1,3434 \text { à } 20^{\circ} \mathrm{C} . \\
\mathrm{R}_{0}=1,3350 \quad \text { id. }
\end{array}\right\} \text { Sensibilité }=0,0084 \text { environ }
$$

D'après L. Panchaud, le sérum chloruromercurique des laits acides est toujours clair et sa réfraction diminue, parce que l'acide lactique formé aux dépens du lactose a une réfraction plus faible que le lactose.

Le sérum chloruromercurique contenant de l'acide chlorhydrique libre, celui-ci libère la chaux de la caséine, de sorte que l'influence de l'acide lactique qui aurait pu être partiellement salifié par de la chaux se fait directement sentir.

Le sérum chloruromercurique des laits malades est généralement clair, mais il donne un trouble par addition de réactif chloruromercurique.

Critique: Avantages: Lacto-sérum toujours très clair, sans albumines, de filtration relativement rapide. Economie de chauffage et de temps.

Inconvénients: Prix élevé du réactif. Coagulant dangereux (toxique et caustique).

\section{B. Lacto-sérums préparés a chaud.}

\section{Sérums à l'acide acétique.}

Nous nous bornerons à rappeler ici à propos de ces sérums, ce que nous avons dit au cours de l'historique de la réfractométrie des lactosérums. Les auteurs qui ont préconisé l'emploi de l'acide acétique comme coagulant, utilisent en général une solution à 20 ou à $25 \%$ à raison de $2 \mathrm{~cm}^{3}$ de cette solution par $100 \mathrm{~cm}^{3}$ de lait. La température à laquelle 
est porté le mélangẽ et la durée d'échauffement varient avec les auteurs ; si bien que, suivant les procédés employés, l'albumine coagulable reste en totalité ou disparaît en partie, apportant ainsi à l'indice de réfraction une augmentation plus ou moins forte.

Nous ne retiendrons ici que la méthode préconisée par HENSEvaL et Mulute.

Mode opératoire: $50 \mathrm{~cm}^{3}$ de lait sont additionnés de $1 \mathrm{~cm}^{3}$ d'acide acétique à $20 \%$, puis chauffés au bain-marie à $65^{\circ}-70^{\circ}$ pendant 5 minutes, en ayant soin de ne pas dépasser cette dernière température; on refroidit ensuite vers $15^{\circ}$, on filtre et on examine au réfractomètre :

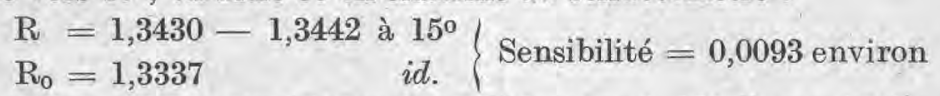

Pour les auteurs un lait est mouillé lorsque à $15^{\circ} \mathrm{C}$. son indice de réfraction est inférieur à 1,3425 ; il est suspect de 1,3425 à 1,3429 .

L'usage simultané, sur un même lait, des tétrasérums I et II de Pfyl et TuRnau nous a permis, en nous basant sur la différence entre les indices fournis par le tétrasérum I, contenant les albumines et le tétrasérum II n'en renfermant pas, de constater que l'augmentation d'indice, provoqué par les albumines, varie entre 0,0003 et 0,0012 .

Le sérum de Henseval et Mulare renferme les albumines et l'indice, entre leur maximum et leur minimum peut baisser, sans pour cela qu'il y ait eu mouillage, de 0,0009 .

Critique: Avantages: Nuls.

Inconvénients : Lacto-sérum trouble dans 1 'immense majorité des cas et d'une longueur de filtration désespérante dans tous les cas. Nous avons été souvent dans l'impossibilité de déterminer l'indice, par suite du trouble du filtrat, même en changeant le filtre à plusieurs reprises. Enfin la présence d'albumines doit faire rejeter ces sérums de la pratique courante.

\section{Tétrasérum II}

\section{(Pfyl et Turnau.)}

Mode opératoire: On chauffe le lait pendant 20 minutes, au bainmarie bouillant, dans un ballon muni d'un tube à reflux. Après refroidissement, on rejette dans le ballon où se trouve le lait l'eau condensée dans le tube refroidisseur. On traite ensuite $50 \mathrm{~cm}^{3}$ de lait refroidi dans les mêmes conditions que pour la fabrication du Tétrasérum I.

Le sérum est clair jusqu'à $16^{\circ}$ S.H. d'acidité du lait ${ }^{1}$.

Critique: Ce sérum est trop long à préparer pour être pratiquement utilisable dans la recherche du mouillage en série. Il peut servir très efficacement, par comparaison avec le Tétrasérum $I$, à reconnaître si le lait a été chauffé ou additionné de lait bouilli.

1 Les degrés d'acidité Soxhlex-Henkex donnent le nombre de centimètres cubes de soude $1 / 4$ normale saturant l'acidité de $100 \mathrm{em}^{3}$ de lait. 


\title{
$3^{\circ}$ Sérum chlorurocalcique
}

\author{
(E. Ackermann.)
}

Préparation de la solution coagulante: On dissout $200 \mathrm{gr}$. de chlorure de calcium fondu dans l'eau distillée de manière à obtenir 1 litre de solution. Le poids spécifique de la solution doit être de 1,1375 à $15^{\circ} \mathrm{C}$.; diluée à $1 / 10$, elle doit donner à la température de $17^{\circ} 5 \mathrm{C}$. un indice de réfraction de 1,3374 .

Mode opératoire: $30 \mathrm{~cm}^{3}$ de lait, contenus dans un gros tube à essais, sont additionnés de $0,25 \mathrm{~cm}^{3}$ de la solution de chlorure de calcium. On agite vivement, on munit le gros tube d'un réfrigérant et on plonge dans un bain-marie en pleine ébullition, On maintient l'ébullition vive pendant 15 minutes, puis on refroidit dans un courant d'eau froide. On mélange ensuite l'eau de condensation déposée dans les parois du tube à essais et du réfrigérant avec le reste du sérum, en retournant le tube à essais ; on décante ensuite le sérum et on détermine l'indice de réfraction à $17^{\circ} 5 \mathrm{C}$.

Jusqu'à $9^{\circ}$ S.H. d'acidité du lait, le sérum est clair, il se trouble audessus de cette limite et à partir de $12^{\circ} \mathrm{S}$.H. une filtration devient nécessaire pour pouvoir procéder à l'examen réfractométrique.

$\mathrm{R}$ varie entre 1,3422 et 1,3429 à $17^{\circ}, 5 \mathrm{C}$. | Sensibilité $=0,0088$

$\mathrm{R}^{\circ}=1,3336 \quad i d . \quad$ environ.

Critiques: Avantages: Préparation du sérum simple et rapide. Pas de filtration.

Inconvénients: Avec les laits acides, le sérum est trouble et la réfraction s'élève de 0,0003 à 0,0008 ce qui peut dissimuler un mouillage faible.

Rappelons que pour L. Panchaud, eette élévation de l'indice de réfraction est due au lactate de chaux, formé par saturation de la chaux de la caséine, par l'acide lactique de fermentation et aux peptones, d'origine bactérienne, qui ne présipitent pas par le chlorure de calcium.

Pour les laits malades (mammites), qui sont alcalins, le sérum est également trouble (les albumines n'étant pas toutes précipitées par le chlorure de calcium) et la réfraction basse.

La cause du trouble d'un lacto-sérum chlorurocalcique sera vite reconnue en faisant l'essai à l'alcool-alizarine : les laits acides coagulent en jaune, les laits alcalins coagulent, ou non, en violet.

Le trouble qui se produit dans le sérum ehlorurocalcique est done toujours l'indice d'une anomalie, il est souvent un indicateur précieux, qui permet de déterminer l'origine du lait examiné.

\section{CHOIX D'UNE MÉTHODE.}

Il convient tout d'abord de remarquer que toutes les méthodes décrites précédemment impliquent une filtration du lacto-sérum. Seule 'la méthode au chlorure de calcium d'Ackermann fait exception à la 
règle. Le sérum, avec cette méthode, peut être examiné par simple décantation, tant est compact le coagulum formé par le chlorure de calcium.

Or, le fait de pouvoir se passer de filtration pour l'examen réfractométrique est de la plus haute importance.Dans les examens en séries, comportant jusqu'à 50 échantillons, il implique un gain de temps considérable et diminue, dans de grandes mesures, les chances d'erreur.

Notons aussi que tous les lacto-sérums préparés à froid (sauf le sérum chloruromereurique de Ambuhl et Weiss) contiennent encore lactalbumine et lactoglobuline. Or, comme nous l'avons vu, ces albumines sont en proportions relativement variables dans le lait. Lorsqu'un lacto-sérum les renferme, sa réfraction perd, de ce fait, son caractère de constance qui lui confère toute sa valeur scientifique et pratique.

Seules, deux méthodes de production retiendront done notre attention :

\section{La méthode chlorurocalcique d'Ackermann,}

La méthode chloruromercurique d'Ambuhl et Weiss.

Nous n'hésitons pas à donner la préférence à là méthode chloruroealcique. Elle a donné lieu, depuis 1907 , à un nambre si considérable d'essais et à une étude tellement approfondie, qu'on peut dire qu'elle est la mieux connue. Le procédé d'Ackermann a fait ses preuves et est employé, sur une grande échelle, dans les laboratoires allemands et suisses où il donne entière satisfaction.

Grâce aux travaux de G. Wiegner et Ackermann, il est facile, connaissant l'indice de réfraction du sérum chlorurocalcique, d'en déduire le poids spécifique de ce sérum et de calculer le résidu sec maigre du lait examiné, toutes applications de la méthode dont il convient d'ap. précier la portée pratique.

Dans les cas exceptionnels où les laits auraient un degré d'acidité trop élevé et donneraient, même après filtration du sérum chlorurocalcique, un trouble trop considérable pour pouvoir être examinés au réfractomètre, il conviendrait d'employer, avec ces laits, la méthode chloruromercurique qui donne un sérum clair, quel que soit le degré d'acidité des laits.

Les deux méthodes, loin de s'exclure, peuvent donc s'employer de front dans ces cas bien définis.

\section{Influence des substances conservatrices ajoutées au lait sur l'indice de réfraction des lacto-sérums chlorurocalciques}

Il arrive souvent que les échantillons de lait prélevés en vue de la recherche des fraudes ne sont pas soumis immédiatement à l'examen du laboratoire. Dans ces conditions, il est de règle d'ajouter au lait une substance conservatrice. La plus employée est le bichromate de potassium.

II résulte des recherches de E. Mollennaugr que l'addition à 
$100 \mathrm{~cm}^{3}$ de lait d'un centimètre cube de solution de bichromate de potassium, ayant un poids spécifique de 1,3 (soit à peu près celui du lait) est sans influence sur l'indice de réfraction. Le fait d'ajouter cette substance au lait permet sa conservation pendant un minimum de 48 heures.

1 décigramme d'aldéhyde formique, ajouté à $100 \mathrm{~cm}^{3}$ de lait, ne modifie pas la réfraction de son sérum chlorurocalcique et permet la conservation de l'échantillon en attendant son examen réfractométrique.

\section{E. Utilisation de la réfractométrie pour la recherche rapide du mouillage.}

Puisque nous avons fait choix de la méthode d'Ackermann au chlorure de calcium, nous allons voir dans quelle mesure ce procédé est susceptible de rendre des services dans la recherche rapide, en série, du mouillage des laits.

Rappelons tout.d'abord que l'indice de réfraction du sérum est complètement indépendant de la teneur du lait en matière grasse, ainsi qu'il résulte des expériences de MaI et Rothenfusser. Un défaut de brassage du lait, au moment du prélèvement, n'empêchera donc pas la recherche réfractométrique du mouillage.

Pour le démontrer, Mai et Rothenfusser introduisent deux litres de lait dans un récipient muni d'un robinet à sa partie supérieure.

Ils abandonnent le lait au repos pendant quelques heures et soutirent ensuite un litre par le robinet :

\begin{tabular}{lcc} 
Lait primitif $\ldots \ldots \ldots \ldots \ldots \ldots \ldots \ldots$ & $\begin{array}{c}\text { Réfraction } \\
1,3426\end{array}$ & $\begin{array}{c}\text { Matière grasse } \\
\text { Lait (portion inférieure) } \ldots \ldots \ldots \ldots \ldots\end{array}$ \\
Lait (portion supérieure) & 1,3426 & $4,1 \% \ldots \ldots$ \\
\hline
\end{tabular}

Deux laits de traite (matin et soir) donnent le même indice de réfraction.

La réfraction reste constante, même si l'on observe des variations dans le chiffre du résidu sec maigre.

La traite incomplète, qui influe sur la quantité de matière grasse, est sans action sur la réfraction.

Lo changement de nourriture, qui cause des variations dans la teneur en graisse, n'a qu'une influence minime sur la réfraction.

Les laits des vaches fraîchement vélées ne se distinguent pas des laits normaux, quant à la réfraction; la lactation n'a pas d'influence importante.

Comme nous l'avons vu, Ackermann donne pour les laits normaux non falsifiés, des indices de lacto-sérum compris entre 1,3422 et 1,3429 à 1705 .

Il sera facile à un expert, appelé à travailler journellement sur les 
laits de sa région, d'établir des chiffres limites pour l'indice des lactosérums chlorurocalciques.

Nous ne pouvons mieux faire que de rappeler ici, à ce propos, les remarques de Mai et Rothenfusser :

"Pour le lait, encore moins que pour les autres produits naturels, on ne peut fixer des chiffres limites absolus. A cette occasion, nous devons dire que nous tenons la fixation d'un minimum, quant à lä teneur en graisse du lait, pour une absurdité.

"On ne doit jamais comparer les laits à un lait normal, mais bien à un lait moyen de composition fixée par une longue expérience dans la même contrée. Dans ces conditions seulement, les chiffres limites ont leur raison d'être.

"L'expert doit voir dans chaque échantillon de lait qui lui est soumis une véritable personnalité, qu'il doit juger pour elle-même en la comparant avec des types analogues et en tenant compte de toutes les circonstances dans lesquelles il a été prélevé.

"De tous les chiffres analytiques, c'est la réfraction qui varie le moins, même après des périodes assez longues. C'est cette constance de la réfraction qui donne une grande valeur à la méthode. »

Dans les cas de réfraction basse, il sera bon de procéder à une contreépreuve à l'étable ou au lieu de ramassage des laits pour se faire une opinion, car juger suspect de mouillage sans contre-épreuve un lait est une opération risquée, pour de faibles mouillages (jusqu’à $5 \%$ ), tout au moins.

La méthode réfractométrique n'a de valeur que pour les laits de mélange, elle est souvent en défaut quand il s'agit de laits individuels dont la composition est soumise à de grandes variations.

Pour calculer la proportion d'eau ajoutée au lait, on peut utiliser avec avantage des tables analogues à celle que nous reproduisons, d'après MaI et Rothenfusser ; on la détermine par rapport au degré $39(1,3424)$ de réfraction.

Lorsque les contre-épreuves donnent des réfractions différentes de 1,3424 , on peut calculer les valeurs correspondantes par interpolation.

Le procédé chlorurocalcique permet à un praticien habile de préparer en deux heures 50 sérums et de déterminer leur indice de réfraction, à condition d'employer la cuve d'Ackermann avec son support pour éprouvettes et le réfractomètre à immersion de Zeiss.

Le procédé est donc rapide. Est-il suffisant à lui "seul pour faire la preuve du mouillage des laits? Nous sommes obligé de convenir que la méthode ne se suffit pas à elle-même et que, dans aucun cas, elle ne permet d'affirmer le mouillage, quand il s'agit de l'établir formellement. Pas plus que les autres méthodes physiques : cryoscopie, viscosimétrie, mesure de la résistivité électrique, la détermination de l'indice de réfraction ne permet de se dispenser de l'analyse. 
TABLE POUR LE CALOUL DU MOUHLAGE (Sérum chlorurocalcique) établie pour le chiffre $39 \mathrm{R} . \mathrm{Z} .\left(n_{d}=1,3424\right)$ (d'après MAI \& RothenfusseR)

\begin{tabular}{|c|c|c|c|c|c|c|c|c|}
\hline $\begin{array}{c}\text { Divisions } \\
\text { réfraetom. } \\
\text { Zeiss } \\
\end{array}$ & $n_{d}$ & $\begin{array}{c}\% / 0 \\
\text { d'eau }\end{array}$ & \begin{tabular}{||c|} 
Divisions \\
réfractom. \\
Zeiss
\end{tabular} & $n_{d}$ & $\begin{array}{c}\% \\
\text { d'eau }\end{array}$ & $\begin{array}{c}\text { Divisions } \\
\text { réfractom. } \\
\text { Zeiss }\end{array}$ & $n_{d}$ & $\begin{array}{c}\% \\
\text { d'eau }\end{array}$ \\
\hline 37,9 & 1,3419 & 4 & 34,8 & 1,34078 & 20 & 32,5 & 1,33991 & 36 \\
\hline 37,7 & 1,34187 & 5 & 34,6 & 1,3407 & 21 & 32,4 & 1,3398 & 37 \\
\hline 37,5 & 1,3418 & 6 & 34,4 & 1,3406 & 22 & 32,2 & 1,33979 & 38 \\
\hline 37,3 & 1,3417 & 7 & 34,3 & 1,34059 & 23 & 32,1 & 1,33975 & 39 \\
\hline 37,1 & 1,3416 & 8 & 34,2 & 1,3405 & 24 & 32 & 1,33972 & 40 \\
\hline 36,9 & 1,34158 & 9 & 34 & 1,34048 & 25 & 31,9 & 1,33968 & 41 \\
\hline 36,7 & 1,3415 & 10 & 33,9 & 1,34044 & 26 & 31,8 & 1,33964 & 42 \\
\hline 36,5 & 1,3414 & 11 & 33,7 & 1,34036 & 27 & 31,6 & 1,33956 & 43 \\
\hline 36,3 & 1,3413 & 12 & 33,6 & 1,34032 & 28 & 31,5 & 1,33953 & 44 \\
\hline 36,1 & 1,34127 & 13 & 33,4 & 1,34025 & 29 & 31,4 & 1,33949 & 45 \\
\hline 35,9 & 1,3412 & 14 & 33,3 & 1,34021 & 30 & 31,3 & 1,33945 & 46 \\
\hline 35,7 & 1,3411 & 15 & 33,2 & 1,34017 & 31 & 31,2 & 1,3394 & 47 \\
\hline 35,5 & 1,34105 & 16 & 33 & 1,3401 & 32 & 31,1 & 1,33937 & 48 \\
\hline 35,3 & 1,3409 & 17 & 32,9 & 1,3400 & 33 & 31 & 1,33934 & 49 \\
\hline 35,1 & 1,34089 & 18 & 32,7 & 1,33998 & 34 & 30,9 & 1,3393 & 50 \\
\hline 35 & 1,3408 & 19 & 32,6 & 1,33994 & 35 & & & \\
\hline
\end{tabular}

Dans les cas de doute, si aucune contre-épreuve n'est possible, on pourra déterminer, parallèlement à l'indice de réfraction, le point de congélation du lait ou sa constante moléculaire simplifiée (C.M.S.).

Avec une réfraction basse, une C.M.S. normale et un point de congélation normal, on aura affaire à un lait non mouillé ; au contraire, avec une réfraction basse, une C.M.S. basse et un point de congélation élevé, on pourra conclure formellement au mouillage du lait.

Les méthodes réfractométriques sont donc d'excellentes méthodes de triage. Elles permettent, dans un laps de temps très restreint, de contrôler un grand nombre d'échantillons de lait et par conséquent de dépister la fraude avec une singulière facilité.

Quand un échantillon de lait a un indice de réfraction supérieur au chiffre limite admis pour la méthode utilisée et pour la région où l'on opère, il n'est pas mouillé. L'indice de réfraction et la matière grasse du lait donnent une image suffisante de cette sécrétion pour permettre de supprimer la détermination de la densité et le calcul du résidu sec maigre, d'où, pour les laboratoires, un gain de temps très appréciable.

Enfin, rappelons qu'avec le sérum chlorurocalcique, pour ceux qui ne disposent pas d'un réfractomètre, il est possible de se contenter de la détermination de la densité ou du résidu sec du sérum, des tables fournissant l'équivalence entre la réfraction, la densité et le résidu sec. 
Lorsqu'il s'agit de laits frais, normaux, on peut déduire de la réfraction du sérum ehlorurocalcique : le lactose, les cendrés et même les protéiques globaux du lait. Cette opération revient à exprimer la réfraction du lacto-sérum, en unités de ces différents éléments, en se basant, bien entendu, sur la composition normale type du sérum d'un lait de composition moyenne et de remplir ainsi facilement les colonnes d'un bulletin d'analyse. Ces déductions sont fausses pour les laits anormaux, aigres, très gras et malades ${ }^{1}$. Enfin, dans les cas où le mouillage est soupçonné, le sérum chlorurocalcique peut être utilisé pour la recherche des nitrates.

Les méthodes réfractométriques ont reçu une consécration officielle en Allemagne et en Suisse où elles sont employées, d'une façon courante, à la grande satisfaction des laboratoires qui les utilisent.

II serait à souhaiter qu'elles fussent introduites dans nos laboratoires de contrôle des denrées alimentaires, où la rapidité de leur exécution rendrait possible l'examen quotidien d'un plus grand nombre d'échantillons de lait et permettrait ainsi de poursuivre les fraudeurs avec une rigueur qu'il ne connaissent pas jusqu'à présent.

On est étonné, en effet, de constater le nombre vraiment insuffisant des prélèvements de laits faits en vue de la recherche des fraudes.

Dans le Département de la Côte-d'Or, pendant l'année 1927, il a été prélevé seulement 303 échantillons de lait et sur ce nombre 52 échantillons ont été reconnus falsifiés, soit une moyenne de $17,16 \%$, ayant entraîné 20 condamnations devant les tribunaux.

Ces chiffres ont leur éloquence. Ils montrent que la Loi du $1^{\text {er }}$ août 1905 sur la répression des fraudes est pratiquement inopérante, puisque les fraudeurs ont la certitude presque absolue de demeurer impunis. Une méthode de tirage rapide des laits, comme la réfractométrie, serait appelée, croyons-nous, à permettre de multiplier très largement le nombre de prélèvements de lait et cela sans grever le budget des laboratoires.

\section{APPENDICE}

\section{RÉFRACTOMÈTRES EMPLOYÉS.}

Pour faire nos mesures d'indices sur les lacto-sérums d'ultra-filtration que nous obtenions en faible quantité, nous a vons employé le réfractomètre de Abbe (Zeiss à Iéna). Cet appareil nous permettait en effet d'opérer sur une goutte de liquide. Il est d'un maniement facile, encore que la détermination de la quatrième déeimale de l'indice se fasse à l'estime.

Pour nos synthèses de lacto-sérums et nos recherches sur les différents lacto-sérums, nous avons utilisé le réfractomètre de Ch. FERY. Pour la pratique courante des laboratoires de recherches sur le lait, nous eonseillons l'emploi du réfractomètre à immersion de ZEIss. Cet

1 D'après L. Panohaud. 
appareil est d'une grande précision, d'un maniement simple et rapide et d'un nettoyage facile; il permet d'opérer sur plusieurs échantillons de sérums, contenus dans de petits béchers et maintenus à la même température dans une cuve d'eau à chauffage constant.

Dans la pratique, les lectures de l'échelle oculaire remplaçent complètement les indices. Une table permet de transformer ces déviations empiriques en indices de réfraction (nous avons opéré toutes ces transformations au cours de notre travail).

D'après SIDERSKY ${ }^{1}$, la méthode d'immersion présente deux avantages : $1^{0}$ elle permet de déterminer la réfraction d'un liquide aussi simplement qu'on mesure sa température au moyen du thermomètre. $2^{\circ}$ Le procédé donne une limite bien plus nette que celui utilisant une couche de liquide emprisonnée entre deux prismes, ce qui permet d'augmenter le grossissement de la lunette d'observation et d'atteindre ainsi une plus haute précision.

Ce réfractomètre, très employé en Allemagne et en Suisse, serait à introduire en France pour l'examen des sérums du lait. Nous l'avons manipulé au Laboratoire Cantonal de Genève, où $M$. le $D^{r}$ PANCHAUD, qui l'utilise journellement depuis de nombreuses années, nous en a recommandé chaudement l'usage.

\section{CONCLUSIONS}

De notre "Contribution a L'ÉTUde DE LA RÉfRAotométrie DES LACTO-SÉRUMS, " nous nous croyons autorisé à tirer les conclusions suivantes :

$1^{0}$ Le lacto-sérum idéal sera celui qui ne renfermera pas de substances protéiques et dont le volume et la composition se rapprocheront le plus de la phase cristalloïde du lait, que les travaux de Connalba, Wiegner et Ch. Porcher ont montré être la plus constante des différentes phases - émulsoïde et colloïdale - de cette liqueur.

$2^{\circ}$ Nous avons obtenu ce lacto-sérum idéal par ultrafiltration du lait.

$3^{\circ}$ Par la méthode graphique - qui permet de faire abstraction de l'augmentation apportée à l'indice de réfraction par le coagulant employé - nous avons retrouvé l'indice absolu $\mathrm{N}_{0}$ du lacto-sérum ultrafiltré pour les sérums chlorurocalcique et chloruromercurique, libres tous deux de substances protéiques.

$4^{\circ}$ L'obtention du lacto-sérum d'ultrafiltration étant très longue, son utilisation ne saurait être recommandée dans la pratique courante.

Son étude nous a permis de constater que pour des laits de mélange de la région de Dijon, son indice de réfraction, $\mathrm{N}_{0}$ varie de 1,3415 à 1,3424 ( $t=20^{\circ}$ C.).

$5^{\circ}$ Des résultats de la synthèse de dix échantillons du lacto-sérum

${ }^{1}$ D. SIDERSKY : La réfractométrie et ses applications pratiques. Gauthier-Villars, Editeurs, Paris. 
d'ultrafiltration, basée sur les travaux de Ch. Porcher et Chevaluier, et faite après l'analyse des laits expérimentés, nous avons pu déduire la moyenne suivante, quant aux augmentations que chacun de ses composants apporte à l'indice de réfraction :

L'ensemble des matières salines augmente l'indice de réfraction de l'eau distillée $(n=1,3330)$ de 0,0014 environ.

Le lactose augmente l'indice (eau distillée + matières salines) de 0,0073 environ.

Parmi les matières salines, celles qui ont le plus d'influence sur la rófraction du lacto-sérum sont, par ordre de grandeur décroissante : les citrates, le chlorure de sodium, les phosphates et en dernier lieu le bicar. bonate de sodium, le sulfate de potassium et l'urée.

Le lactose joue le rôle prineipal dans l'augmentation apportée à l'indice (plus des 5/6).

Nous concluons done que déterminer l'indice de réfraction d'un lactosérum c'est évaluer, en quelque sorte, la teneur du lait en lactose.

$6^{\circ}$ De l'étude comparative des divers modes de production des lactosérums, il résulte que seules les méthodes donnant des sérums libres d'albumines sont à retenir.

Le meilleur procédé sera celui qui remplira le mieux les conditions suivantes :

a) Précipiter toute l'albumine du lait ;

b) Employer un coagulant qui augmente le moins possible l'indice de réfraction du sérum ;

c) Diluer le lait au minimum par le volume utilisé de solution coagulante ;

d) Avoir une sensibilité maximum pour la recherche du mouillage.

$7^{\circ}$ Parmi les procédés de préparation des lacto-sérums nous avons retenu, comme devant être utilisée dans la pratique courante, la méthode d'ACKERMANN, au chlorure de calcium.

Ce procédé est rapide, peu coûteux et le sérum, libre d'albumines, peut être examiné au réfractomètre par simple décantation, ce qui implique un gain de temps considérable.

L'indice du sérum chlorurocalcique se rapproche beaucoup de celui du sérum d'ultrafiltration, il ne le dépasse que de 0,0003 .

$8^{\circ}$ Pour les laits acides, qui donnent des sérums troubles avec la méthode d'ACKERMANN, on peut utiliser avec profitla méthode chloruromercurique de АмвUнL et Werss qui fournit des sérums clairs, quel que soit le degré d'acidité du lait, et dont la sensibilité, en ce qui concerne la recherche du mouillage, est du même ordre de grandeur que celle du sérum chlorurocalcique.

$9^{\circ}$ L'appareil de choix pour l'examen réfractométrique des lactosérums est le réfractomètre à immersion de Zeiss. Il est d'une grande précision, d'un maniement simple et rapide et son nettoyage est aisé; 
enfin, il permet, grâee à la disposition de la cuve qui lui est annexée, d'opérer simultanément sur un grand nombre de sérums maintenus à la même température.

$10^{\circ} \mathrm{La}$ réfractométrie est une méthode sûre et rapide de triage des laits. Elle n'a de valeur que pour les laits de mélange, la composition des laits individuels étant soumise à de trop larges variations.

Dans tous les cas où l'indice de réfraction d'un lacto-sérum est inférieur au chiffre limite adopté pour la région où l'on opère, la méthode réfractométrique doit être étayée par une contre-épreuve à l'étable qui lèvera tous les doutes.

Dans le cas où cette contre-épreuve ne pourrait être faite, les résultats de l'examen réfractométrique seront comparés aux données fournies par la eryoscopie ou par la C.M.S.

$11^{0}$ Nous souhaitons que le procédé réfractométrique - qui depuis de longues années a acquis droit de cité en Allemagne et en Suisse soit adopté en France.

Il permettrait, croyons-nous, par la rapidité de son exécution, de multiplier le nombre véritablement insuffisant des prélèvements de lait faits en vue de la recherche des fraudes et contribuerait ainsi à rendre véritablement opérant, en ce qui concerne le lait et les produits de laiterie, le Décret du 25 mars 1924 portant règlement d'Administration publique pour l'application de la loi du $1^{\text {er }}$ août 1905 sur la répression des fraudes.

(Fin).

\section{BIBLIOGRAPHIE ANALYTIQUE}

\section{$1^{\circ}$ JOURNAUX, REVUES, SOGIETÉS SAVANTES PRODUCTION - HYGIÈNE}

Grunewald Max. - Traitement hygiénique du lait. Svenska Mejeritidningen, 19e année, No 51, 17 décembre 1927, p. 413-415.

Le $38^{\mathrm{e}}$ Congrès Médical allemand tenu à Innsbruck en automne 1924 avait émis le vœu que le contrôle du commerce du lait, qui est moins satisfaisant que celui de la viande, soit étendu aux snimaux et aux étables. Ce vœu était justifié. L'épidémie allemande de fièvre typhoïde de l'été 1925 fut surtout causée par le lait. Aussi, dès 1926, des mesures furent prises par le Ministère de l'Hygiène Prussien pour fəire eontrôler les laiteries centrales et les compagnies des eaux.

La ville de Mannheim a une fourniture de lait tout à fait hygiénique. Une seule Société s'en occupe : $83 \%$ de son eapital appertiennent à la ville.

Les résultats sont les suivants :

En 1912-1914, $14 \%$ des échantillons prélevés étaient falsifiés et $20 \%$ contenaient trop d'impuretés ; en 1924, 0,75\% seulement étaient falsifiés et, en 1925, $0,12 \%$ seulement. Aucun échantillon ne contenait trop d'impuretés ; en 1912-1913, la teneur moyenne du lait en matière grasse était de 3,40\%. En 1924, elle était de $3,86 \%$.

C. Wolf. 